Теорія Ймовір. та Матем. Статист. Вип. 74, 2006
Theor. Probability and Math. Statist.

No. 74, 2007, Pages 77-91

S 0094-9000(07)00699-0

Article electronically published on June 29, 2007

\title{
ORDINAL LAW OF THE ITERATED LOGARITHM IN BANACH LATTICES AND SOME APPLICATIONS
}

UDC 519.21

\author{
I. K. MATSAK
}

\begin{abstract}
Necessary and sufficient conditions are found for the ordinal law of the iterated logarithm in Banach lattices of type $L^{p}$. As a corollary of our general results, we obtain a new law of the iterated logarithm for empirical processes in the spaces $L^{p}(-\infty, \infty)$.
\end{abstract}

\section{INTRODUCTION}

Let $\xi, \xi_{1}, \xi_{2}, \ldots$ be independent identically distributed random variables such that $\mathrm{E} \xi=0$ and $\mathrm{E} \xi^{2}=\sigma^{2}$. Then the random variable $\xi$ satisfies the law of the iterated logarithm, that is,

$$
\lim _{n \rightarrow \infty} \sup \frac{\sum_{i=1}^{n} \xi_{i}}{\chi(n)}=\sigma, \quad \lim _{n \rightarrow \infty} \inf \frac{\sum_{i=1}^{n} \xi_{i}}{\chi(n)}=-\sigma \quad \text { almost surely }
$$

where $\chi(t)=(2 t L L(t))^{1 / 2}$ and $L(t)=\max (1, \ln (t))$ for $t>0$.

The law of the iterated logarithm (11) for the Bernoulli trials is proved by Khintchin [1] (in the framework of his studies in number theory). The next step is done by Kolmogorov [2], who found a fundamental theorem on the law of the iterated logarithm for the general case of independent random variable (not necessarily identically distributed). Equalities (11) for independent identically distributed random variables are proved in [3].

The law of the iterated logarithm is studied in Banach spaces, too. Usually the socalled bounded and compact law of the iterated logarithms are proved in the case of Banach spaces. Below is the underlying definition.

Let $X$ be a random element assuming values in a separable Banach space $B,\left(X_{i}\right)$ a sequence of independent copies of $X$, and $S_{n}=\sum_{i=1}^{n} X_{i}$. We say that the random element $X$ satisfies the bounded law of the iterated logarithm if

$$
\limsup _{n \rightarrow \infty} \frac{\left\|X_{1}+\cdots+X_{n}\right\|}{\chi(n)}<\infty \quad \text { almost surely. }
$$

Similarly, we say that the random element $X$ satisfies the compact law of the iterated logarithm if there exists a compact symmetric set $K$ in $B$ such that

$$
\lim _{n \rightarrow \infty} d\left(\frac{X_{1}+\cdots+X_{n}}{\chi(n)}, K\right)=0
$$

and

$$
\lim _{n \rightarrow \infty} p t\left(\frac{X_{1}+\cdots+X_{n}}{\chi(n)}\right)=K
$$

2000 Mathematics Subject Classification. Primary 60B12.

Key words and phrases. Law of the iterated logarithm, Banach lattices, empirical processes. 
almost surely where $d(x, K)=\inf \{\|x-y\|: y \in K\}$ and $\lim p t\left(x_{n}\right)$ is the set of limit points of the sequence $\left(x_{n}\right)$. Note that $K=K_{X}$ is the unit closed ball of the Hilbert space $H_{R}$ in $B$ associated with the covariance operator $R$ of the random element $X$ (see, for example, 4]).

The main results concerning the bounded and compact laws of the iterated logarithm can be found in [4].

There is a comprehensive literature on the law of the iterated logarithm. We mention the books [4] and [5] and the paper [6], to name a few, where one can find surveys on the law of the iterated logarithm.

The main aim of this paper is to study a new variant of the law of the iterated logarithm, called the ordinal law of the iterated logarithm, for random elements assuming values in a Banach lattice. The definition of the ordinal law of the iterated logarithm is introduced in [7. We need some notions of the theory of Banach lattices (see [8]-[10]) to recall the definition of the ordinal law of the iterated logarithm.

In what follows, $B$ denotes a separable Banach lattice equipped with the norm $\|\cdot\|$ and modulus $|\cdot|$. A subset $A$ of a Banach lattice $B$ is called ordinally bounded if there are two elements $y$ and $z$ such that $y \leq x \leq z$ for all $x \in A$. A Banach lattice $B$ is called $\sigma$-complete if the infimum and supremum exist for any ordinally bounded sequence $x_{n} \in B$. The infimum and supremum are defined for an ordinally bounded sequence $\left(x_{n}\right)$ in a $\sigma$-complete Banach lattice as

$$
\limsup _{n \rightarrow \infty} x_{n}=\inf _{m}\left(\sup _{n \geq m} x_{n}\right) \text { and } \liminf _{n \rightarrow \infty} x_{n}=\sup _{m}\left(\inf _{n \geq m} x_{n}\right),
$$

respectively (see [8]).

We say that a Banach lattice $B$ is ordinally continuous if

$$
0 \leq x_{\alpha} \downarrow 0 \Rightarrow\left\|x_{\alpha}\right\| \rightarrow 0 .
$$

A $q$-concave Banach lattice is an important example of a $\sigma$-complete ordinally continuous lattice ([9, p. 49]).

One can define the operation $\left(\sum_{n=1}^{n}\left|x_{i}\right|^{p}\right)^{1 / p}$ for all elements $x_{1}, x_{2}, \ldots, x_{n}$ in a Banach lattice $B$ (see [9, pp. 40-42]). Thus one can introduce the following definition.

Let $1 \leq p<\infty$. A Banach lattice $B$ is called an $p$-convex if there exists a constant $D^{(p)}=D^{(p)}(B)$ such that

$$
\left\|\left(\sum_{n=1}^{n}\left|x_{i}\right|^{p}\right)^{1 / p}\right\| \leq D^{(p)}\left(\sum_{n=1}^{n}\left\|x_{i}\right\|^{p}\right)^{1 / p}
$$

for all $n$ and all elements $\left(x_{i}\right)_{1}^{n} \subset B$. Accordingly, a Banach lattice $B$ is called $p$-concave if there exists a constant $D_{(q)}=D_{(q)}(B)$ such that

$$
\left(\sum_{n=1}^{n}\left\|x_{i}\right\|^{q}\right)^{1 / q} \leq D_{(q)}\left\|\left(\sum_{n=1}^{n}\left|x_{i}\right|^{q}\right)^{1 / q}\right\|
$$

for all $n$ and all elements $\left(x_{i}\right)_{1}^{n} \subset B$. Below we recall the definition of the mean $\psi$ deviation of a random variable and its generalization to the case of random elements in a Banach lattice (see [7, 11]).

A convex, even, positive for $t \neq 0$, continuous function $\psi(t)$ defined on $R^{1}$ is called an $N$-function ([10]) if $\lim _{t \rightarrow 0} t^{-1} \psi(t)=0$ and $\lim _{t \rightarrow \infty} t^{-1} \psi(t)=+\infty$. Given an $N$-function $\psi(t)$, we define its conjugate $N$-function by

$$
\psi^{*}(t)=\sup _{s \in R^{1}}(s t-\psi(s)) .
$$


Let a random element $X$ assume values in a separable Banach lattice $B$ and let $\psi(t)$ be an $N$-function. Then $\mathfrak{S}_{\psi} X=\sup \left(x \in K_{\psi}(X)\right)$ is called the mean $\psi$-deviation of the random element $X$ where

$$
K_{\psi}(X)=\left\{\mathbf{E}(\eta X): \eta \text { a random variable such that } \mathbf{E} \psi^{*}(\eta) \leq 1\right\}
$$

and $\mathbf{E}$ denotes the Pettis integral.

According to this definition, the mean deviation of order $p, 1<p<\infty$, of a random element $X$ is defined by $\mathfrak{S}_{p} X=\sup \left(x \in K_{p}(X)\right)$ where $K_{p}(X)=\left\{\mathbf{E}(\eta X): \mathbf{E}|\eta|^{q} \leq 1\right\}$, $1 / p+1 / q=1$. In particular, the mean square deviation is $\mathfrak{S} X=\mathfrak{S}_{2} X$.

The mean square deviation $\mathfrak{S} X$ coincides with the classical mean square deviation for a random variable $X$ in the space $R^{1}$ with $\mathbf{E} X=0$ and with the pointwise deviation $\left(\mathbf{E}|X(t)|^{2}\right)^{1 / 2}, t \in T$, for an ideal Banach space on a measurable space $(T, \Lambda, \mu)$.

Let $X$ be a random element defined on a probability space $(\Omega, \Sigma, \mathrm{P})$ that assumes values in a separable Banach space $B$ and such that $\mathbf{E} X=0$. We say that the random element $X$ satisfies the ordinal law of the iterated logarithm if

$$
\limsup _{n \rightarrow \infty} \frac{S_{n}}{\chi(n)}=\mathfrak{S} X, \quad \liminf _{n \rightarrow \infty} \frac{S_{n}}{\chi(n)}=-\mathfrak{S} X \quad \text { almost surely. }
$$

If a Banach lattice $B$ is ordinally continuous, then we obtain from (2) that

$$
\lim _{m \rightarrow \infty}\left\|\sup _{n>m} \frac{S_{n}}{\chi(n)}-\mathfrak{S} X\right\|=0, \quad \lim _{m \rightarrow \infty}\left\|\inf _{n>m} \frac{S_{n}}{\chi(n)}+\mathfrak{S} X\right\|=0 \quad \text { almost surely. }
$$

The inverse implication (3) $\Rightarrow(2)$ holds for Banach lattices being of the type $C(Q)$. Therefore we use (3) as the definition of the ordinal law of the iterated logarithm for such lattices.

We also note that there is a big difference between the bounded (or compact) law of the iterated logarithm and ordinal law of the iterated logarithm in the general case. For example, there is a random element $X$ in the space $l_{2}$ such that

$$
\mathrm{E}\|X\|_{l_{2}}^{2}=\|\mathfrak{S} X\|_{l_{2}}^{2}<\infty .
$$

Thus both bounded and compact laws of the iterated logarithm hold but the ordinal law of the iterated logarithm does not [7].

The following sufficient condition for the ordinal law of the iterated logarithm (2) is found in [7] for $q$-concave $(q<\infty)$ Banach lattices:

$$
\mathfrak{S}_{\phi}(X) \text { exists }
$$

where

$$
\phi(t)= \begin{cases}|t|^{2}, & \text { for } 1 \leq q<2 \\ \frac{|t|^{2} \ln \left(1+|t|^{2}\right)}{L L\left(1+|t|^{2}\right)}, & \text { for } q=2, \\ |t|^{q}, & \text { for } 2<q<\infty\end{cases}
$$

(also see [12]).

Moreover, the results of [7] and [12] imply for $q$-concave Banach lattices that if condition (4) holds, then

$$
\lim _{m \rightarrow \infty} \mathbf{E}\left\|\sup _{n>m} \frac{S_{n}}{\chi(n)}-\mathfrak{S} X\right\|^{q}=0, \quad \lim _{m \rightarrow \infty} \mathbf{E}\left\|\inf _{n>m} \frac{S_{n}}{\chi(n)}+\mathfrak{S} X\right\|^{q}=0 .
$$

Condition (4) is necessary for (5) in the case of the spaces $L^{q}$ and $l_{q}$.

The moment condition (4) seems to be quite restrictive and one does not expect it to be necessary for the ordinal law of the iterated logarithm (2) in the general case. Nevertheless this is not always true, as we show in Section 2 below. It turns out that condition (4) is necessary and sufficient for the ordinal law of the iterated logarithm (2) 
for $q$-concave Banach lattices if $1 \leq q<2$. An analogous assertion for $q=2$ holds under the extra assumption (8). However condition (44) can be improved, indeed, in the case of $q>2$.

In Section 3 we consider some applications of the results obtained in Section 2 to empirical processes.

\section{NECESSARY AND SUFFICIENT CONDITIONS FOR THE ORDINAL LAW OF THE ITERATED LOGARITHM IN SPACES OF THE TYPE $L^{p}$}

Theorem 2.1. Let $B$ be a separable $q$-concave Banach lattice, $1 \leq q<2$, and let $X$ be a random element assuming values in $B$ and such that $\mathrm{E} X=0$. Then the random element $X$ satisfies the ordinal law of the iterated logarithm (2) if and only if $\mathfrak{S} X$ exists in B. If the ordinal law of the iterated logarithm (2) holds, then

$$
C_{1}\|\mathfrak{S} X\| \leq \mathfrak{S}_{q}\left(\left\|\sup _{n \geq 1} \frac{\left|S_{n}\right|}{\chi(n)}\right\|\right) \leq C_{2}\|\mathfrak{S} X\| .
$$

Theorem 2.2. Let $B$ be a separable $q$-concave and $p$-convex Banach lattice, $2<p \leq$ $q<\infty$, and let $X$ be a random element in $B$ such that $\mathbf{E} X=0$. If

$$
\mathbf{E}\|X\|^{2}<\infty
$$

then the random element $X$ satisfies the ordinal law of the iterated logarithm (2).

Theorem 2.3. Let $B$ be a separable $\sigma$-complete Banach lattice and let $X$ be a random element in $B$ such that $\mathbf{E} X=0$. Then

(i) if $B$ is a 2-concave Banach lattice and condition (41) holds for $\phi(t)=\frac{|t|^{2} \ln \left(1+|t|^{2}\right)}{L L\left(1+|t|^{2}\right)}$, then the random element $X$ satisfies the ordinal law of the iterated logarithm (2);

(ii) if $B$ is a 2-convex Banach lattice and

$$
\mathbf{E}\|X\|^{2+\epsilon}<\infty
$$

for some $\epsilon>0$, then condition (44) for $\phi(t)=\frac{|t|^{2} \ln \left(1+|t|^{2}\right)}{L L\left(1+|t|^{2}\right)}$ is necessary and sufficient for the ordinal law of the iterated logarithm (2).

It is known 9] that the space $L^{p}(\mu), 1 \leq p<\infty$, is a $q$-concave Banach lattice if $q \geq p$, while it is an $r$-convex Banach lattice if $1 \leq r \leq p$. This observation together with Theorem 2.3 implies

Corollary 2.1. If $X$ is a random element in the space $L^{2}$ (or in $l_{2}$ ) and condition (8)

holds, then condition (4) for $\phi(t)=\frac{|t|^{2} \ln \left(1+|t|^{2}\right)}{L L\left(1+|t|^{2}\right)}$ is necessary and sufficient for the ordinal law of the iterated logarithm (2).

Remark 2.1. It is clear that condition (44) for $\phi(t)=\frac{|t|^{2} \ln \left(1+|t|^{2}\right)}{L L\left(1+|t|^{2}\right)}$ is not necessary for the ordinal law of the iterated logarithm (2) in the space $l_{2}$ if one does not impose an extra condition like (8). Indeed, consider a simple example:

$$
X=\xi \cdot x, \quad x \in l_{2}, \quad \mathbf{E} \xi=0, \mathbf{E} \xi^{2}<\infty, \mathbf{E} \phi(\xi)=\infty .
$$

Remark 2.2. Any separable $\sigma$-complete Banach lattice is isomorphic to some ideal Banach space ([9, p. 25]). This isomorphism preserves the order and supremums (infimums). Using this isomorphism, one can reduce the proof of many results for abstract Banach lattices to the partial case of the ideal Banach space on a measurable space $(T, \Lambda, \mu)$ such that $\mu(T)=1$. 
Proof of Theorem 2.1. Sufficiency. The law of the iterated logarithm (2) and the second inequality in (6) are proved in [7] for the case where the mean square deviation $\mathfrak{S} X$ of the random element $X$ exists in the Banach lattice $B$.

Necessity. According to Remark 2.2 one needs to consider the case where $B$ is the ideal Banach space on the measurable space $(T, \Lambda, \mu)$. If the law of the iterated logarithm (2) holds, then there exists the random element $\sup _{n \geq 1}\left|S_{n}\right| / \chi(n)$ in the space $B$. We know from the Strassen theorem [13] (proved for $R^{1}$ ) that

$$
\sup _{n \geq 1} \frac{\left|S_{n}(t)\right|}{\chi(n)} \geq \lim _{n \rightarrow \infty} \sup \frac{\left|S_{n}(t)\right|}{\chi(n)} \geq \mathfrak{S}(X(t)) \quad \text { almost surely }
$$

almost everywhere on $T$, or, equivalently, that

$$
\mu\left(t \in T: \sup _{n \geq 1} \frac{\left|S_{n}(t)\right|}{\chi(n)} \geq \mathfrak{S}(X(t))\right)=1 \quad \text { almost surely. }
$$

This means for the ideal Banach space $B$ that $\mathfrak{S} X=\{\mathfrak{S} X(t), t \in T\}$ exists in $B$ and the first inequality in (6) holds.

Proof of Theorem 2.2. We prove the first equality in (2). It is equivalent to the relation

$$
\mathrm{o}_{m \rightarrow \infty} \sup _{n \geq m} \frac{S_{n}}{\chi(n)}=\mathfrak{S} X \quad \text { almost surely }
$$

(here o- $\lim _{n \rightarrow \infty} x_{n}=x$ denotes the ordinal convergence (o-convergence) of a sequence of elements $\left(x_{n}\right)$ to an element $\left.x[8,10]\right)$.

As in the proof of Theorem 2.1 we assume that $B$ is the ideal Banach space on the measurable space $(T, \Lambda, \mu), X=\{X(t), t \in T\}, \mathfrak{S} X=\{\sigma(t), t \in T\}$, and $\mu(T)=1$.

It is known [10] that relation (9) follows from

$$
\begin{gathered}
\mu\left(t \in T: \limsup _{n \rightarrow \infty} \frac{S_{n}(t)}{\chi(n)}=\sigma(t)\right)=1 \quad \text { almost surely, } \\
\left\|\sup _{n \geq 1} \frac{\left|S_{n}\right|}{\chi(n)}\right\|<\infty \quad \text { almost surely }
\end{gathered}
$$

if the ideal Banach space is separable and $\sigma$-complete.

Since a $p$-convex Banach lattice is 2-convex for $p>2$ [9], we have

$$
\left(\mathbf{E}\|X\|^{2}\right)^{1 / 2} \geq C(B)\|\mathfrak{S} X\|
$$

(see [1]). Therefore we proved the implication (7) $\Rightarrow \exists \mathfrak{S} X$.

This result and the law of the iterated logarithm for independent identically distributed random variables in $R^{1}$ implies that

$$
\limsup _{n \rightarrow \infty} \frac{S_{n}(t)}{\chi(n)}=\sigma(t) \quad \text { almost surely }
$$

almost everywhere on $T$. It remains to apply the Fubini theorem to complete the proof of equality (10).

The proof of (11) is a more complicated problem. First we assume that $X$ is a symmetric random element. Then one can assume that $X=\epsilon \hat{X}$ where $\hat{X}$ and $\epsilon$ are independent, $\hat{X}$ is a copy of $X$, while $\epsilon$ is a symmetric Bernoulli random variable.

Let $\left(X_{n}\right)$ be a sequence of independent copies of $X$. Put

$$
\begin{array}{cl}
\bar{X}_{n}=\hat{X}_{n} I\left(\left\|\hat{X}_{n}\right\| \leq \sqrt{n}\right), \quad \tilde{X}_{n}=\hat{X}_{n} I\left(\left\|\hat{X}_{n}\right\|>\sqrt{n}\right), \\
\bar{S}_{n}=\sum_{i=1}^{n} \epsilon_{i} \bar{X}_{i}, \quad \tilde{S}_{n}=\sum_{i=1}^{n} \epsilon_{i} \tilde{X}_{i} .
\end{array}
$$


It is clear that $X_{n}=\epsilon_{n}\left(\bar{X}_{n}+\tilde{X}_{n}\right)$ and

$$
\left\|\sup _{n \geq 1} \frac{\left|S_{n}\right|}{\chi(n)}\right\| \leq\left\|\sup _{n \geq 1} \frac{\left|\bar{S}_{n}\right|}{\chi(n)}\right\|+\left\|\sup _{n \geq 1} \frac{\left|\tilde{S}_{n}\right|}{\chi(n)}\right\| \quad \text { almost surely. }
$$

We know that condition (7) implies that

$$
\sum_{n \geq 1} \mathrm{P}\left(\left\|X_{n}\right\| \geq \sqrt{n}\right)<\infty
$$

(see [14]). Now the Borel-Cantelli lemma proves that only a finite number of random elements $\tilde{X}_{n}$ differ from zero almost surely, that is,

$$
\left\|\sup _{n \geq 1} \frac{\left|\tilde{S}_{n}\right|}{\chi(n)}\right\|<\infty \quad \text { almost surely. }
$$

Therefore inequality (11) follows if the first term on the right hand side of (12) is bounded. To show its boundedness, we prove that

$$
\mathbf{E}_{\hat{X}}\left\|\sup _{n \geq 1} \frac{\left|\bar{S}_{n}\right|}{\chi(n)}\right\|^{q}<\infty \quad \text { almost surely }
$$

where $\mathbf{E}_{\hat{X}}(\xi)$ denotes the expectation of the random variable $\xi$ given the sequence $\left(\hat{X}_{n}\right)$.

We need the following auxiliary result to prove (13).

Lemma 2.1. Let $\left(\xi_{i}\right)$ be a sequence of independent random variables such that $\mathbf{E} \xi_{i}=0$. Then for all $p>0$

$$
\mathbf{E}\left(\sup _{n \geq 1} \frac{\left|\xi_{1}+\xi_{2}+\cdots+\xi_{n}\right|}{\chi(n)}\right)^{p} \leq C_{p} \mathbf{E}\left(\sup _{n \geq 1} \frac{\xi_{1}^{2}+\xi_{2}^{2}+\cdots+\xi_{n}^{2}}{n}\right)^{p / 2} .
$$

The proof of Lemma 2.1 can be found in 7) (similar moment bounds for the law of the iterated logarithm are considered in [15]).

Along with Lemma 2.1 we use another bound of the paper [11]:

$$
\left(\mathrm{E}\|Y\|^{q}\right)^{1 / q} \leq D_{(q)}\left\|\mathfrak{S}_{q}(Y)\right\|
$$

where $Y$ is a random element assuming values in a $q$-concave Banach lattice and $D_{(q)}$ is the constant involved in the definition of a $q$-concave Banach lattice. Bounds (14) and (15) imply that

$$
\begin{aligned}
& \left(\left.\mathbf{E}_{\hat{X}}\left\|\sup _{n \geq 1} \frac{\left|\bar{S}_{n}\right|}{\chi(n)}\right\|\right|^{q}\right)^{1 / q} \leq D_{(q)}\left\|\left(\mathbf{E}_{\hat{X}} \sup _{n \geq 1}\left|\frac{\bar{S}_{n}(t)}{\chi(n)}\right|^{q}\right)^{1 / q}\right\| \\
& \leq C_{q} D_{(q)}\left\|\left(\sup _{n \geq 1} \frac{\sum_{i=1}^{n} \bar{X}_{i}^{2}(t)}{n}\right)^{1 / 2}\right\| .
\end{aligned}
$$

Next we pass from the Banach lattice $B$ to the so-called 2-convexification $B_{(2)}$ of $B$. We describe briefly this construction (see [9, p. 53] for details). For $B_{(2)}$ we take $B$ with the original order and introduce operations

$$
x \oplus y=\left(x^{2}+y^{2}\right)^{1 / 2}, \quad \alpha \odot x=\alpha^{1 / 2} x .
$$

For $\alpha^{2}$ we take $\operatorname{sign}(\alpha)|\alpha|^{2}$ and define $\left(x^{2}+y^{2}\right)^{1 / 2}$ as an element of $B$ that corresponds to the function $f(s, t)=\operatorname{sign}\left(s^{2}+t^{2}\right)\left|s^{2}+t^{2}\right|^{1 / 2}([9, \mathrm{p} .53])$. The number

$$
\||x|\|=\inf \left(\sum_{1}^{n}\left\|x_{i}\right\|^{2}:|x|=\sum_{1}^{n} \oplus\left|x_{i}\right|, x_{i} \in B, n \geq 1\right)
$$


is the lattice norm in the space $B_{(2)}$ if the space $B$ is 2-convex. Moreover

$$
\||| x|| \leq\| x\left\|^{2} \leq\left(D^{(2)}\right)^{2}\right\| x \| \mid
$$

for all $x \in B$ where $D^{(2)}$ is the constant involved in the definition of the 2-convexity.

Taking into account both inequalities in (17), the right hand side of (16) can be estimated from above by

$$
\left\|\left(\sup _{n \geq 1} \frac{\sum_{i=1}^{n} \bar{X}_{i}^{2}}{n}\right)^{1 / 2}\right\| \leq D^{(2)}\left\|\sup _{n \geq 1} \frac{1}{n} \odot\left(\bar{X}_{1} \oplus \bar{X}_{2} \oplus \cdots \oplus \bar{X}_{n}\right)\right\| \|^{1 / 2} .
$$

The last step of the proof in the symmetric case is based on the following auxiliary result.

Lemma 2.2. Let $V$ be a separable $q$-concave and $p$-convex Banach lattice,

$$
1<p \leq 2 \leq q<\infty
$$

and let $Y, Y_{1}, Y_{2}, \ldots$ be independent identically distributed random elements assuming values in $V$. Then

$$
\left(\mathbf{E}\left\|\sup _{n \geq 1} \frac{\left|\sum_{i=1}^{n} Y_{i} I\left(\left\|Y_{i}\right\| \leq i\right)\right|}{n}\right\|^{p}\right)^{1 / p} \leq\|\mathbf{E}|Y|\|+C(B)(\mathbf{E}\|Y\|)^{1 / p} .
$$

An implicit form of Lemma 2.2 can be found in the proof of Theorem 1 of [16.

Since the Banach lattice $B$ is $q$-concave and $p$-convex, $2<p \leq q<\infty$, the lattice $B_{(2)}$ is $q / 2$-concave and $(p / 2)$-convex $(9$, p. 54]). Without loss of generality one can assume that $2<p \leq 4$, in which case the lattice $B_{(2)}$ satisfies the conditions of Lemma 2.2 .

According to Lemma 2.2 and bounds (16) and (18) we have

$$
\begin{aligned}
\mathbf{E}\left\|\sup _{n \geq 1} \frac{\left|\bar{S}_{n}\right|}{\chi(n)}\right\|^{p} & \leq\left. C(B) \mathbf{E}\left\|\sup _{n \geq 1} \frac{1}{n} \odot\left(\bar{X}_{1} \oplus \bar{X}_{2} \oplus \cdots \oplus \bar{X}_{n}\right)\right\|\right|^{p / 2} \\
& \left.\leq C(B)(\|\mathfrak{S} X\|\|+\mathbf{E}\| X \|)^{2 / p}\right)^{p / 2} \\
& \leq C(B)\left(\|\mathfrak{S} X\|^{p}+\mathbf{E}\|X\|^{2}\right)<\infty .
\end{aligned}
$$

Therefore bounds (13) and (11) are proved for symmetric random elements.

We reduce the general case to the symmetric case by using the standard symmetrization procedure. Let

$$
\begin{gathered}
\bar{X}_{n}=X_{n} I\left(\left\|X_{n}\right\| \leq \sqrt{n}\right), \quad \bar{X}_{n}^{\prime}=X_{n}^{\prime} I\left(\left\|X_{n}^{\prime}\right\| \leq \sqrt{n}\right), \\
\bar{X}_{n}^{(s)}=\bar{X}_{n}-\bar{X}_{n}^{\prime}, \quad \bar{S}_{n}^{(s)}=\sum_{i=1}^{n} \bar{X}_{i}^{(s)}
\end{gathered}
$$

where $\left(X_{n}^{\prime}\right)$ is an independent copy of the sequence $\left(X_{n}\right)$.

Similarly to the symmetric case, the main point of the proof in the general case is to check inequality (13).

Random elements $\bar{X}_{n}^{(s)}$ are symmetric and satisfy inequality (19). Applying a well known moment bound in Banach spaces ([17, p. 222]), we obtain

$$
\mathbf{E}\left\|\sup _{n \geq 1} \frac{\left|\bar{S}_{n}-\sum_{k=1}^{n} \mathbf{E} \bar{X}_{k}\right|}{\chi(n)}\right\|^{p} \leq \mathbf{E}\left\|\sup _{n \geq 1} \frac{\left|\bar{S}_{n}^{(s)}\right|}{\chi(n)}\right\|^{p}<\infty .
$$

It remains to prove

$$
\left\|\sup _{n \geq 1} \frac{\left|\sum_{k=1}^{n} \mathbf{E} \bar{X}_{k}\right|}{\chi(n)}\right\|<\infty
$$


Recall that $\mathbf{E} X=0$ by assumption. Thus

$$
\begin{aligned}
\mathbf{E} \bar{X}_{n}(t) & =\mathbf{E} X(t) I(\|X\|>\sqrt{n}) \\
& \leq\left(\mathbf{E}|X(t)|^{2}\right)^{1 / 2}(\mathrm{P}(\|X\|>\sqrt{n}))^{1 / 2} \\
& \leq\left(\mathbf{E}\|X\|^{2}\right)^{1 / 2} \sigma(t) / \sqrt{n},
\end{aligned}
$$

whence

$$
\left\|\sup _{n \geq 1} \frac{\left|\sum_{k=1}^{n} \mathbf{E} \bar{X}_{k}\right|}{\chi(n)}\right\| \leq C\left(\mathbf{E}\|X\|^{2}\right)^{1 / 2}\|\mathfrak{S} X\| .
$$

As noted above, $\|\mathfrak{S} X\|<\infty$ and therefore inequality (20) is proved.

Remark 2.3. It is known for Banach spaces of type 2 that (4 p. 216]) a random element $X$ satisfies the bounded law of the iterated logarithm if and only if

$$
\mathbf{E} \frac{\|X\|^{2}}{L L(X)}<\infty
$$

and

$$
\mathbf{E} f X=0, \quad \mathbf{E} f^{2} X<\infty
$$

for all $f \in B^{\prime}$. Thus (21) is a necessary condition for the law of the iterated logarithm (2), too.

Another necessary condition is that $\mathfrak{S} X$ exists (this follows from the proof of Theorem 2.1 where we do not use the $q$-concavity in the necessity part). A natural question arises on whether or not condition (17) can be replaced in Theorem 2.2 by the set of conditions: $\mathfrak{S} X$ exists and (21) holds.

An answer to this question is not yet known to the author.

Proof of Theorem 2.3. Since (i) is proved in [7] and [12, we show that (ii) holds. First we prove that condition (ii) implies

$$
\mathbf{E}\left\|\sup _{n \geq 1} \frac{\left|X_{n}\right|}{\chi(n)}\right\|^{2}<\infty .
$$

The obvious inequality

$$
\frac{\left|X_{n}\right|}{\chi(n)} \leq \frac{\left|S_{n}\right|}{\chi(n)}+\frac{\left|S_{n-1}\right|}{\chi(n-1)} \quad \text { almost surely }
$$

and the law of the iterated logarithm (2) yield

$$
\|M\|<\infty \quad \text { almost surely }
$$

where

$$
M=\sup _{1 \leq n \leq \infty} \frac{\left|X_{n}\right|}{\chi(n)}<\infty .
$$

Now we apply a modification of the Yurinskiu method [18 for sums of independent random elements in Banach spaces. For the 2-convexification $B_{(2)}$ equipped with the norm $\||\cdot| \mid$ we introduce the following notation:

$$
\begin{gathered}
M_{n}=\sup _{1 \leq k \leq n} \frac{\left|X_{k}\right|}{\chi(k)}, \quad M_{n, i}=\sup _{1 \leq k \leq n, k \neq i} \frac{\left|X_{k}\right|}{\chi(k)}, \\
\zeta_{i}=\mathbf{E}_{i}\left\|M_{n}\right\|-\mathbf{E}_{i-1} \mid\left\|M_{n}\right\|, \\
\mathbf{E}_{i} \eta=\mathbf{E}\left(\eta / F_{i}\right)
\end{gathered}
$$

where $F_{i}=\sigma\left(X_{1}, \ldots, X_{i}\right)$ is the $\sigma$-algebra generated by the random elements $X_{1}, \ldots, X_{i}$ and $F_{0}$ is the trivial $\sigma$-algebra. 
Then we have the following martingale representation

$$
\left\|M_{n}\right\||| \mathbf{E}|| M_{n} \| \mid=\sum_{i=1}^{n} \zeta_{i}
$$

Using the representation

$$
\zeta_{i}=\left(\mathbf{E}_{i}-\mathbf{E}_{i-1}\right)\left(\left\|M_{n}\right\||-|\left\|M_{n, i}\right\|\right),
$$

bound (17), and the inequality

$$
\left\|M_{n}\left|\|-\| M_{n, i}\right|\right\|\left|\left\|\frac{X_{i}}{\chi(i)} \mid\right\|\right.
$$

we obtain

$$
\left|\zeta_{i}\right| \leq\left|\left\|\frac{X_{i}}{\chi(i)}\right\|\right|+\mathbf{E}\left\|\frac{X_{i}}{\chi(i)}\right\| \mid \leq \frac{\left\|X_{i}\right\|^{2}+\mathbf{E}\left\|X_{i}\right\|^{2}}{2 i L L(i)} .
$$

Since $\left(\zeta_{i}\right)$ is a martingale-difference, bounds (8) and (25) for $1<r=1+\epsilon / 2 \leq 2$ imply that

$$
\mathbf{E}\left|\sum_{i=1}^{n} \zeta_{i}\right|^{r} \leq 2 \sum_{i=1}^{n} \mathbf{E}\left|\zeta_{i}\right|^{r} \leq C_{r} \mathbf{E}\left\|X_{i}\right\|^{2 r} \sum_{i=1}^{\infty}(i L L(i))^{-r}<\infty
$$

(see [19]). The latter inequality and representation (24) allow us to conclude that the sequence of random variables $\left(\left\|M_{n}\right\|\|-\mathbf{E}\|\left|M_{n} \|\right|\right)$ is stochastically bounded, that is,

$$
\lim _{C \rightarrow \infty} \sup _{n \geq 1} \mathrm{P}\left(|||| M_{n}|||-\mathbf{E}||| M_{n}||||>C\right)=0 .
$$

The Banach lattice $B$ is separable and $\sigma$-complete under the assumptions of Theorem 2.3. thus it is ordinally continuous ([9, p. 7]). Using bound (23), we get

$$
\text { ||| } M_{n}|| \uparrow|||M||| \quad \text { almost surely }
$$

as $n \rightarrow \infty$. Now the Fatou theorem implies that

$$
\mathbf{E}\left\|M_{n}\right\||\uparrow \mathbf{E}\||M|\| \text {. }
$$

Were inequality (22) wrong, we would have

$$
\mathbf{E}\|M\|=\infty .
$$

Therefore

$$
\left\|\left|M_{n}\|\mid-\mathbf{E}\| M_{n} \| \stackrel{\mathrm{P}}{\longrightarrow} \infty,\right.\right.
$$

contradicting (26). This proves inequality (22).

If inequality (22) holds, then condition (44) is easy to check by using the results of the papers 11] and 12. Indeed

$$
\infty>\left(\mathbf{E}\left\|\sup _{n \geq 1} \frac{\left|X_{n}\right|}{\chi(n)}\right\|^{2}\right)^{1 / 2} \geq C_{1}\left\|\mathfrak{S}\left(\sup _{n \geq 1} \frac{\left|X_{n}\right|}{\chi(n)}\right)\right\| \geq C_{2}\left\|\mathfrak{S}_{\phi}(X)\right\| .
$$

Related to Theorem 2.3 is a question on whether or not a moment condition like (8) is sufficient for the ordinal law of the iterated logarithm (2) in the space $l_{2}$.

The following example gives a negative answer to this question.

Example 2.1. Let a random element $X$ assume values in the space in $l_{2}$ and let it be such that

$$
\mathbf{E}\|X\|_{l_{2}}^{m}<\infty
$$


for all $m>1$. Let

$$
\left\|\sup _{n \geq 1}\left|\frac{X_{n}}{\chi(n)}\right|\right\|_{l_{2}}=\infty \quad \text { almost surely }
$$

and let $\left(X_{n}\right)$ be a sequence of independent copies of the random element $X$.

Note that the ordinal law of the iterated logarithm (2) does not hold for this random element $X$.

The example below is a slight modification of an example in the paper [16. Put

$$
\theta=\sum_{k \geq 1} \frac{1}{k L^{2}(k)}, \quad p_{k}=\frac{1}{\theta k L^{2}(k)}, \quad k \geq 1 .
$$

It is clear that $\sum_{k \geq 1} p_{k}=1$.

Let $\left(\xi_{k}\right)$ be a sequence of random variables such that

$$
\mathrm{P}\left(\xi_{k}=+1\right)=p_{k} / 2, \quad \mathrm{P}\left(\xi_{k}=-1\right)=p_{k} / 2, \quad \mathrm{P}\left(\xi_{k}=0\right)=1-p_{k} .
$$

Then the random element $X=\left(\xi_{k}\right)$ belongs to the space $l_{2}$ and satisfies the conditions

$$
\mathbf{E}\left|\xi_{k}\right|=p_{k}, \quad \mathbf{E}\|X\|_{l_{2}}^{2}=\sum_{k \geq 1} p_{k}=1 .
$$

To show that condition (27) holds for the random element $X$, one has to repeat the corresponding proof of [16].

Equality (28) is checked in the same way as in [16] and is reduced to the proof of the divergence of the equivalent series

$$
\sum_{k \geq 1}\left|\mathfrak{S}_{\phi}\left(\left|\xi_{k}\right|\right)\right|^{2} \sim \sum_{k \geq 1}\left\|\xi_{k}\right\|_{\phi 1}^{2}
$$

where $\|\eta\|_{\phi, 1}=\inf (\lambda>0: \mathbf{E} \phi(\eta / \lambda) \leq 1)$ and $\phi(s)$ is defined in Theorem 2.3.

The norm $\left\|\xi_{k}\right\|_{\phi, 1}$ can be explicitly estimated from below by the definition of the random variable $\xi_{k}$. Indeed

$$
\left\|\xi_{k}\right\|_{\phi, 1}=\inf \left(\lambda>0: \frac{L\left(1+\lambda^{-2}\right)}{\lambda^{2} L L\left(1+\lambda^{-2}\right)} \frac{1}{\theta k L^{2}(k)} \leq 1\right) .
$$

Choosing $\lambda=\left(\theta k L(k) L L\left(k^{2}\right)\right)^{-1 / 2}$, we obtain

$$
\frac{L\left(1+\lambda^{-2}\right)}{\lambda^{2} L L\left(1+\lambda^{-2}\right)} \frac{1}{\theta k L^{2}(k)} \geq 1
$$

for sufficiently large $k>k_{0}$. Thus, for $k>k_{0}$,

$$
\left\|\xi_{k}\right\|_{\phi, 1} \geq \frac{1}{\sqrt{\theta k L(k) L L\left(k^{2}\right)}}
$$

and the series (30) diverge.

\section{Applications to empirical processes}

Let $\xi, \xi_{1}, \xi_{2} \ldots$ be independent identically distributed random variables in $R^{1}$ whose common distribution function is $F(t)$. Consider the empirical distribution function constructed from the sample $\xi_{1}, \ldots, \xi_{n}$ :

$$
F_{n}(t)=\frac{1}{n} \sum_{i=1}^{n} I_{(-\infty, t]}\left(\xi_{i}\right), \quad-\infty<t<\infty .
$$


According to the Glivenko-Cantelli theorem

$$
\sup _{-\infty<t<\infty}\left|F_{n}(t)-F(t)\right| \rightarrow 0 \quad \text { almost surely. }
$$

A substantial improvement of this relation is the law of the iterated logarithm for the empirical distribution function (due to N. V. Smirnov and K. L. Chung; see [20, p. 157]):

$$
\limsup _{n \rightarrow \infty}\left(\frac{2 n}{L L(n)}\right)^{1 / 2} \sup _{-\infty<t<\infty}\left|F_{n}(t)-F(t)\right|=1 \quad \text { almost surely. }
$$

Further investigations of the almost sure asymptotic behavior of the function $F_{n}(t)$ are related to the empirical process

$$
\beta_{n}(t)=\sqrt{n}\left(F_{n}(t)-F(t)\right), \quad-\infty<t<\infty .
$$

Surveys of these investigations can be found in [20] and [21].

Below we derive some new variants of the law of the iterated logarithm for the empirical process $\beta_{n}(t)$ from our results of Section 2. Consider stochastic processes

$$
X_{n}(t)=I_{(-\infty, t]}\left(\xi_{n}\right)-F(t), \quad-\infty<t<\infty .
$$

It is known that

$$
\mathfrak{S}\left(X_{n}\right)=(\sigma(t)=\sqrt{F(t)(1-F(t))},-\infty<t<\infty) .
$$

Then the law of the iterated logarithm (3) for a random element $X_{n}$ assuming values in the space $L^{p}(-\infty, \infty)$ can be rewritten as

$$
\begin{aligned}
& \lim _{m \rightarrow \infty} \int_{-\infty}^{\infty}\left|\sup _{n>m} \frac{\beta_{n}(t)}{\sqrt{2 L L(n)}}-\sqrt{F(t)(1-F(t))}\right|^{p} d t=0 \quad \text { almost surely, } \\
& \lim _{m \rightarrow \infty} \int_{-\infty}^{\infty}\left|\inf _{n>m} \frac{\beta_{n}(t)}{\sqrt{2 L L(n)}}+\sqrt{F(t)(1-F(t))}\right|^{p} d t=0 \quad \text { almost surely. }
\end{aligned}
$$

Corollary 3.1. The empirical process $\beta_{n}(t)$ satisfies the law of the iterated logarithm (31) if one of the following conditions holds:

(i) in the case of $1 \leq p<2$,

$$
\int_{-\infty}^{\infty}|F(t)(1-F(t))|^{p / 2} d t<\infty
$$

(ii) in the case of $p>2$,

$$
\mathbf{E}|\xi|^{2 / p}<\infty
$$

(iii) in the case of $p=2,0<F(t)<1$ for all $t \in(-\infty, \infty)$ and

$$
\int_{0}^{\infty} \frac{(1-F(t)) L(1 /(1-F(t)))}{L L(1 /(1-F(t)))} d t+\int_{-\infty}^{0} \frac{F(t) L(1 / F(t))}{L L(1 / F(t))} d t<\infty .
$$

Moreover condition (32) is necessary for the law of the iterated logarithm (31) in the case of (i).

Corollary 3.2. If $\xi$ is a random variable whose values almost surely belong to the interval $[0,1]$ and $1 \leq p<\infty$, then

$$
\begin{aligned}
& \lim _{m \rightarrow \infty} \int_{0}^{1}\left|\sup _{n>m} \frac{\beta_{n}(t)}{\sqrt{2 L L(n)}}-\sqrt{F(t)(1-F(t))}\right|^{p} d t=0 \quad \text { almost surely, } \\
& \lim _{m \rightarrow \infty} \int_{0}^{1}\left|\inf _{n>m} \frac{\beta_{n}(t)}{\sqrt{2 L L(n)}}+\sqrt{F(t)(1-F(t))}\right|^{p} d t=0 \quad \text { almost surely. }
\end{aligned}
$$


Proof of Corollary 3.1. The space $L^{p}(-\infty, \infty), 1 \leq p<\infty$, is a separable $p$-concave and $p$-convex Banach lattice. Thus (i) is straightforward from Theorem 2.1 by observing that the condition "S $X$ exists" is equivalent to condition (32).

(ii). Let $p>2$ and let condition (33) hold. Then

$$
\begin{aligned}
\mathbf{E}\left\|X_{n}\right\|^{2}= & \mathbf{E}\left(\int_{-\infty}^{\infty}\left(|1-F(t)|^{p} I(\xi<t)+|F(t)|^{p} I(\xi>t)\right) d t\right)^{2 / p} \\
= & \mathbf{E}\left(\int_{\xi}^{\infty}|1-F(t)|^{p} d t+\int_{-\infty}^{\xi}|F(t)|^{p} d t\right)^{2 / p} \\
\leq & \left(\int_{0}^{\infty}|1-F(t)|^{p} d t+\int_{-\infty}^{0}|F(t)|^{p} d t\right)^{2 / p} \\
& +\mathbf{E}\left(\int_{-|\xi|}^{0}|1-F(t)|^{p} d t+\int_{0}^{|\xi|}|F(t)|^{p} d t\right)^{2 / p} \\
\leq & 2 \mathbf{E}|\xi|^{2 / p}+\left(\int_{0}^{\infty}|1-F(t)|^{p} d t\right)^{2 / p}+\left(\int_{-\infty}^{0}|F(t)|^{p} d t\right)^{2 / p} .
\end{aligned}
$$

We noted above that the space $L^{p}(-\infty, \infty)$ for $p>2$ is a 2 -convex Banach lattice. This implies that

$$
\begin{aligned}
\mathbf{E}\left\|X_{n}\right\|^{2} & \geq\left\|\mathfrak{S}\left(X_{n}\right)\right\|^{2}=\left(\int_{-\infty}^{\infty}|F(t)(1-F(t))|^{p / 2} d t\right)^{2 / p} \\
& \geq F(0)\left(\int_{0}^{\infty}|1-F(t)|^{p / 2} d t\right)^{2 / p}+(1-F(0))\left(\int_{-\infty}^{0}|F(t)|^{p / 2} d t\right)^{2 / p} .
\end{aligned}
$$

We choose $d_{\epsilon}>0$ such that $1-F\left(d_{\epsilon}\right) \leq \epsilon$ and $F\left(-d_{\epsilon}\right) \leq \epsilon$. Then

$$
\begin{gathered}
\left(\int_{d_{\epsilon}}^{\infty}|1-F(t)|^{p} d t\right)^{2 / p} \leq \epsilon\left(\int_{d_{\epsilon}}^{\infty}|1-F(t)|^{p / 2} d t\right)^{2 / p}, \\
\left(\int_{-\infty}^{-d_{\epsilon}}|F(t)|^{p} d t\right)^{2 / p} \leq \epsilon\left(\int_{-\infty}^{-d_{\epsilon}}|F(t)|^{p / 2} d t\right)^{2 / p} .
\end{gathered}
$$

An obvious argument proves that

$$
\mathbf{E}|\xi|^{2 / p}<\infty \rightarrow \int_{-\infty}^{\infty}|F(t)(1-F(t))|^{p / 2} d t<\infty
$$

if conditions (35)-(37) hold. Turning to bound (35), we get

$$
\mathbf{E}\left\|X_{n}\right\|^{2}<\infty .
$$

Thus the law of the iterated logarithm (31) follows by Theorem 2.2

(iii). Fix $t$ and estimate $\mathfrak{S}_{\phi}\left(X_{n}(t)\right)$ from above where

$$
\phi(s)=\frac{|s|^{2} \ln \left(1+|s|^{2}\right)}{L L\left(1+|s|^{2}\right)} .
$$

Since $\mathfrak{S}_{\phi}(\cdot)$ is a norm in the Orlicz space of random variables,

$$
\begin{aligned}
\mathfrak{S}_{\phi}\left(X_{n}(t)\right) & =\mathfrak{S}_{\phi}(1-F(t) I(\xi<t)+F(t) I(\xi \geq t)) \\
& \leq(1-F(t)) \mathfrak{S}_{\phi} I(\xi<t)+F(t) \mathfrak{S}_{\phi} I(\xi \geq t) .
\end{aligned}
$$


It is known $([10])$ that the norm $\mathfrak{S}_{\phi}(\eta)$ is equivalent to the norm

$$
\|\eta\|_{\phi, 1}=\inf \left(\lambda>0: \mathbf{E} \phi\left(\frac{\eta}{\lambda}\right) \leq 1\right)
$$

which is easier to estimate in our case.

Indeed

$$
\begin{aligned}
\|I(\xi<t)\|_{\phi, 1} & =\inf \left(\lambda>0: \phi\left(\frac{1}{\lambda}\right) F(t) \leq 1\right) \\
& =\frac{1}{\phi^{-1}(1 / F(t))} .
\end{aligned}
$$

Similarly

$$
\|I(\xi \geq t)\|_{\phi, 1}=\frac{1}{\phi^{-1}(1 /(1-F(t)))} .
$$

Taking into account (38), we get

$$
\mathfrak{S}_{\phi}\left(X_{n}(t)\right) \leq C\left(\frac{1-F(t)}{\phi^{-1}(1 / F(t))}+\frac{F(t)}{\phi^{-1}(1 /(1-F(t)))}\right) .
$$

On the other hand,

$$
\phi^{-1}(t) \sim \sqrt{2 t \frac{\ln \ln t}{\ln t}}, \quad t \rightarrow \infty .
$$

This together with inequality (39) implies that

$$
\mathfrak{S}_{\phi}\left(X_{n}(t)\right) \leq C\left(\frac{(1-F(t)) \sqrt{F(t) \ln (1 / F(t))}}{\sqrt{\ln \ln (1 / F(t))}}+\frac{F(t) \sqrt{(1-F(t)) \ln (1 /(1-F(t)))}}{\sqrt{\ln \ln (1 /(1-F(t)))}}\right)
$$

for large $|t|$. The latter inequality and condition (34) prove that the integral

$$
\int_{-\infty}^{\infty}\left|\mathfrak{S}_{\phi}\left(X_{n}(t)\right)\right|^{2} d t
$$

converges, that is, $\mathfrak{S}_{\phi}\left(X_{n}\right)$ exists in $L^{2}(-\infty, \infty)$.

It remains to apply Theorem 2.3 .

If values of a random variable $\xi$ almost surely belong to the interval $[0,1]$, then conditions (32)-(34) hold. Thus Corollary 3.2 follows from Corollary 3.1 .

Remark 3.1. Let $\xi$ be a random variable uniform on the interval $[0,1]$. Corollary 3.2 implies that the ordinal law of the iterated logarithm (31) holds for the classical $\omega_{n}^{2}$ statistic (with the normalizing function $\psi(t) \equiv 1$ ).

Put

$$
X(t)=\frac{I(\xi<t)-t}{\sqrt{t(1-t)}} .
$$

Then it is easy to check that the ordinal law of the iterated logarithm holds for the weighted statistic $\Omega_{n}^{2}$ (with the normalizing function $\psi(t) \equiv t(1-t)$ ), namely

$$
\lim _{m \rightarrow \infty} \int_{0}^{1}\left|\sup _{n>m} \frac{\beta_{n}(t)}{\sqrt{2 L L(n)}}-\sqrt{t(1-t)}\right|^{2} \frac{d t}{t(1-t)}=0
$$

almost surely. 
It is worth mentioning (see [21]) that the bounded law of the iterated logarithm does not hold for $\Omega_{n}^{2}$ in the uniform metric.

\section{Concluding Remarks}

Necessary and sufficient conditions for the ordinal law of the iterated logarithm (2) are found for $q$-concave Banach lattices. The problem is solved completely for $1 \leq q<2$. The sufficient conditions for $q=2$ are necessary under the extra moment assumption (8). Sufficient condition (7) is stronger than known conditions for the bounded law of the iterated logarithm if $q>2$. It is a matter of further investigation to improve condition (7) for the case of $q>2$.

Applications of the main results of the paper are presented in Section 3 for empirical processes.

\section{BIBLIOGRAPHY}

1. A. Khintchin, Über einen Satz der Wahrscheinlichkeitsrechnung, Fund. Math. 6 (1924), 9-12.

2. A. N. Kolmogorov, Probability Theory and Mathematical Statistics, "Nauka", Moscow, 1986; English transl., Kluwer Academic Publishers Group, Dordrecht 1992. MR861120 (88d:01043) MR:1153022 (92j:01071)

3. P. Hartman and A. Wintner, On the law of the iterated logarithm, Amer. J. Math. 63 (1941), no. 1, 169-176. MR0003497 (2:228h)

4. M. Ledoux and M. Talagrand, Probability in Banach Spaces, Springer, Berlin, 1991. MR.1102015 (93c:60001)

5. V. V. Petrov, Sums of Independent Random Variables, "Nauka", Moscow, 1972; English transl., Springer-Verlag, New York-Heidelberg, 1975. MR0322927 (48:1288) MR0388499 (52:9335)

6. N. H. Bingham, Variants of the law of the iterated logarithm, Bull. London Math. Soc. 18 (1986), 433-467. MR847984 (87k:60087)

7. I. K. Matsak, The law of the iterated logarithm in Banach lattices, Teor. Veroyatnost. Primenen. 44 (1999), no. 4, 865-874; English transl. in Theory Probab. Appl. 44 (2000), 775-784. MR1811138(2003a:60008)

8. K. Yosida, Functional Analysis, Springer-Verlag, Berlin, 1965. MR0180824 (31:5054)

9. J. Lindenstraus and L. Tzafriri, Classical Banach Spaces, vol. 2, Springer, Berlin, 1979. MR 0415253(54:3344)

10. L. V. Kantorovich and G. P. Akilov, Functional Analysis, "Nauka", Moscow, 1984; English transl., Pergamon Press, Oxford-Elmsford, New York, 1982. MR664597 (83h:46002)

11. I. K. Matsak, Mean $\psi$-deviation of a random element in a Banach lattice and its applications, Teor. Imovirnost. Matem. Statist. 60 (1999), 123-135; English transl. in Theory Probab. Mathem. Statist. 60 (2000), 137-149. MR1826150

12. I. K. Matsak, Estimates for the moments of the supremum of normalized sums of independent random variables, Teor. Imovirnost. Matem. Statist. 67 (2002), 104-116; English transl. in Theory Probab. Mathem. Statist. 67 (2003), 115-128. MR 1956624(2004i:60061)

13. V. Strassen, A converse to the law of the iterated logarithm, Z. Wahrscheinlichkeitstheorie verw. Geb. 4 (1966), 265-268. MR0200965 (34:850)

14. W. Feller, An Introduction to Probability Theory and its Applications, vol. II, John Wiley \& Sons, Inc., New York-London-Sydney, 1971. MR0270403 (42:5292)

15. G. Pisier, Sur la loi du logarithme itere dans les espaces de Banach, Lect. Notes Math. 526 (1976), 203-210. MR0501237 (58:18644)

16. I. K. Matsak, Some remarks to the ordinal strong law of large numbers, Teor. Imovirnost. Matem. Statist. 72 (2005), 84-92; English transl. in Theory Probab. Mathem. Statist. 72 (2006), 93-102. MR2168139 (2006f:60011)

17. N. N. Vakhania, V. I. Tarieladze, and S. A. Chobanyan, Probability Distributions on Banach Spaces, "Nauka", Moscow, 1985; English transl., D. Reidel Publishing Co., Dordrecht, 1987. MR.1435288(97k:60007)

18. V. V. Yurinskǐr, Exponential bounds for large deviations, Teor. Veroyatnost. Priminen. XIX (1974), no. 1, 152-153; English transl. in Theory Probab. Appl. XIX (1975), 154-159. MR0334298 (48:12617)

19. B. Bahr and C. G. Essen, Inequalities for the $r$-th absolute moment of a sum of random variables, $1<r<2$, Ann. Math. Statist. 36 (1965), no. 1, 299-303. MR0170407 (30:645) 
20. M. Csörgö and P. Révész, Strong Approximations in Probability and Statistics, Akademiai Kiado, Budapest, 1981. MR.666546 (84d:60050)

21. P. Gaenssler and W. Stout, Empirical processes: a survey of results for independent and identically distributed random variables, Ann. Probab. 7 (1979), no. 2, 193-243. MR.525051 (80d:60002)

Department of Probability Theory and Mathematical Statistics, Faculty for Mathematics and Mechanics, National Taras Shevchenko University, Glushkov Avenue, 6, Kyiv, 03127, UKRAINE

Current address: Kyiv National University for Technology and Design, Nemyrovych-Danchenko Street, 2, 01601, GSP, Kyiv, Ukraine

E-mail address: m_i_k@ukr.net

Received 7/MAY/2004

Translated by OLEG KLESOV 